\title{
An Improved GROMOS96 Force Field for Aliphatic Hydrocarbons in the Condensed Phase
}

\author{
LUKAS D. SCHULER, XAVIER DAURA, WILFRED F. VAN GUNSTEREN \\ Laboratory of Physical Chemistry, Swiss Federal Institute of Technology Zürich, ETH-Zentrum, \\ 8092 Zürich, Switzerland
}

Received 5 February 2001; accepted 19 March 2001

\begin{abstract}
Over the past 4 years the GROMOS96 force field has been successfully used in biomolecular simulations, for example in peptide folding studies and detailed protein investigations, but no applications to lipid systems have been published yet. Here we provide a detailed investigation of aliphatic liquid systems. For liquids of larger aliphatic chains, $n$-heptane and longer, the standard GROMOS96 parameter sets 43A1 and 43A2 yield a too low pressure at the experimental density. Therefore, a reparametrization of the GROMOS96 force field regarding aliphatic carbons was initiated. The new force field parameter set $45 \mathrm{~A} 3$ shows considerable improvements for $n$-alkanes, cyclo-, iso-, and neoalkanes and other branched aliphatics. Liquid densities and heat of vaporization are reproduced for almost all of these molecules. Excellent agreement is found with experiment for the free energy of hydration for alkanes. The GROMOS96 45A3 parameter set should, therefore, be suitable for application to lipid aggregates such as membranes and micelles, for mixed systems of aliphatics with or without water, for polymers, and other apolar systems that may interact with different biomolecules. (C) 2001 John Wiley \& Sons, Inc. J Comput Chem 22: 1205-1218, 2001
\end{abstract}

Keywords: GROMOS96 force field 45A3; aliphatic hydrocarbons; condensed phase properties; molecular dynamics simulation 


\section{Introduction}

I n classical molecular dynamics (MD) simulations the mechanical laws of physics are applied to propagate simulated particles such as atoms and molecules through space and time. Because a fully quantum-mechanical description of the interaction of larger molecules is not feasible, classical MD simulation uses a simpler level of abstraction based on chemical knowledge. Atoms are described as spherically symmetric particles, connected into molecules through covalent bonds and interacting with their noncovalently bound neighbors through van der Waals interactions and, if the interacting particles are charged, through Coulomb-type interactions. The potential energy function that describes the interaction between the particles or atoms is called a force field. ${ }^{1}$ Although most biomolecular force fields are based on a similar functional form of the interaction function, they show considerable differences in their parametrization and parameter values. ${ }^{2-18}$ The GROMOS force field has been developed over many years, ${ }^{10-16}$ and, as with all other force fields, the empirical force-field parameters can only be as satisfactory as comparisons of simulated properties against experimental data demonstrate. ${ }^{19}$ Here we exemplify that an established force field successfully used over the years in biomolecular simulations of a variety of molecules, such as peptides, ${ }^{20-23}$ proteins, ${ }^{24-29}$ sugars, $^{30-32}$ and nucleotides ${ }^{33,34}$ is not necessarily producing satisfactory results for another class of large molecules, simple aliphatic chains. Although a small set of alkanes representative of the types of aliphatic groups present in proteins (with chain lengths smaller than 7) has been successfully parametrized, ${ }^{14,16}$ the same force field yields a too high density (at $1 \mathrm{~atm}$ pressure) or negative pressure (at constant experimental density) when applied to longer alkanes (up to chain length 20). Therefore, a detailed MD investigation of $n$-alkanes and other aliphatic molecules was undertaken and is presented here together with a new set of GROMOS96 force field parameters, called $45 \mathrm{~A} 3$, that is suitable to systems with aliphatic chains of various lengths, such as alkanes, lipids, and polymers.

The most important experimental properties of a liquid to be reproduced in a simulation are the density (volume or pressure), the heat of vaporization, and the free energy of hydration, as a proper description of mixtures with water is essential for most biomolecular systems. These quantities should be in good agreement with experimental data at room temperature, the temperature of most of the applications of the force field. To achieve this goal one must know the influence of the different force-field parameters on the quantities studied. Therefore, the dependence of these quantities upon different force-field parameters, such as van der Waals or Lennard-Jones $C_{12}$ and $C_{6}$ coefficients, nonbonded interaction cutoff length, and charge-group size, is investigated first. The next step is to choose a calibration set of molecules for which the force-field parameters are tuned such that in MD simulations of the condensed phase the experimental density and heat of vaporization are reproduced. Previously, a set of nine linear, branched, and cyclic alkanes of short chain length (up to $6 \mathrm{CH}_{n}$ units) was used to this end. ${ }^{14}$ This set had been chosen with an eye to the occurrence of aliphatic fragments in proteins, sugars, and nucleotides. However, much larger aliphatic chains are present in lipids and membranes. Therefore, the current reparametrization of the aliphatic $\mathrm{CH}_{n}$ atom groups (including $n=0$ ) of the GROMOS96 force field is based on a much larger set of alkanes. For $n$-alkanes, chain lengths up to 20 (eicosane) were considered.

In the reparametrization of the GROMOS96 force field three sets of molecules were distinguished in regard to their properties in the condensed phase as obtained by MD simulation with the GROMOS96 force field: $n$-alkanes, cyclo-alkanes, and branched alkanes such as iso- or neoalkanes. Most common in biomolecular simulations, for example, of lipid membranes, will be chains consisting of $\mathrm{CH}_{2}$ and $\mathrm{CH}_{3}$ atom groups or united atoms. Consistently, in a first parametrization step, the calibration set was composed of $n$-alkanes only. Compared to our previous work $^{14}$ the set of $n$-alkanes was expanded beyond hexane $\left(C_{6}\right)$ to eicosane $\left(C_{20}\right)$.

\section{Methods}

For the parametrization of aliphatics, many simulations had to be carried out. Three types of species were considered: $n$-alkanes, cyclo-alkanes, and branched alkanes (see Tables II-IV). First, liquid systems consisting of 512 individual molecules of the investigated species in a cubic box were built and equilibrated. For all systems the total number of molecules was the same, which made the computational effort rather different for different molecules, depending on their number of atoms and interactions. The molecular topologies were constructed using the GROMOS96 conventions and standard parameter values (see Table I). ${ }^{13,16}$ 
TABLE I.

Force-Field Parameters for Aliphatic Molecules.

\begin{tabular}{|c|c|c|c|c|}
\hline Atom Name & Description & \multicolumn{2}{|c|}{ IAC } & Mass [a.m.u.] \\
\hline $\mathrm{CH}_{1}$ & aliphatic $\mathrm{CH}_{1}$ group & \multicolumn{2}{|c|}{12} & 13.019 \\
\hline $\mathrm{CH}_{2}$ & aliphatic $\mathrm{CH}_{2}$ group & \multicolumn{2}{|c|}{13} & 14.029 \\
\hline $\mathrm{CH}_{3}$ & aliphatic $\mathrm{CH}_{3}$ group & \multicolumn{2}{|c|}{14} & 15.035 \\
\hline $\mathrm{CH}_{4}$ & methane $\mathrm{CH}_{4}$ group & \multicolumn{2}{|c|}{15} & 16.043 \\
\hline $\mathrm{CH}_{0}$ & aliphatic tetrahedral C & \multicolumn{2}{|c|}{45} & 12.011 \\
\hline Bond & Type Code & \multicolumn{2}{|c|}{$b_{0}[\mathrm{~nm}]$} & $K_{\mathrm{b}}\left[\mathrm{kJ} \mathrm{mol}^{-1} \mathrm{~nm}^{-4}\right]$ \\
\hline $\mathrm{CH}_{n}-\mathrm{CH}_{n}$ & 26 & \multicolumn{2}{|c|}{0.153} & $7.15 \times 10^{6}$ \\
\hline Bond Angle & Type Code & \multicolumn{2}{|c|}{$\theta_{0}$ [degree] } & $K_{\theta}\left[\mathrm{kJ} \mathrm{mol}^{-1}\right]$ \\
\hline $\mathrm{CH}_{n}-\mathrm{CH}_{0}-\mathrm{CH}_{n}$ & 12 & \multicolumn{2}{|c|}{109.5} & 520 \\
\hline $\mathrm{CH}_{n}-\mathrm{CH}_{1}-\mathrm{CH}_{n}$ & 12 & \multicolumn{2}{|c|}{109.5} & 520 \\
\hline $\mathrm{CH}_{n}-\mathrm{CH}_{2}-\mathrm{CH}_{n}$ & 14 & \multicolumn{2}{|c|}{111.0} & 530 \\
\hline Improper Dihedral & Type Code & \multicolumn{2}{|c|}{$\xi_{0}$ [degree] } & $K_{\xi}\left[\mathrm{kJ} \mathrm{mol}^{-1}\right.$ degree $\left.^{-2}\right]$ \\
\hline Around $\mathrm{CH}_{1}$ group & 2 & \multicolumn{2}{|c|}{35.26439} & 0.102 \\
\hline Torsional Dihedral & Type & $\cos (\delta)$ & $m_{n}$ & $K_{\varphi}\left[\mathrm{kJ} \mathrm{mol}^{-1}\right]$ \\
\hline $\mathrm{CH}_{n}-\mathrm{CH}_{n}-\mathrm{CH}_{n}-\mathrm{CH}_{n}$ & 17 & +1.0 & 3 & $5.92^{\mathrm{a}}$ \\
\hline
\end{tabular}

The parameters and convertions are defined in ref. 13. The GROMOS96 integer atom code (IAC) describes the van der Waals parameters of the corresponding atoms.

a The value $K_{\varphi}=5.92 \mathrm{~kJ} \mathrm{~mol}^{-1}$ in torsional dihedral type 17 used here is the one from parameter set $43 \mathrm{~A} 2,{ }^{16}$ which is slightly larger than the value $5.86 \mathrm{~kJ} \mathrm{~mol}^{-1}$ of set $43 \mathrm{~A} 1$.

In all simulations the GROMOS96 software ${ }^{13,35}$ was used. A twin-range cutoff scheme with 0.8 and $1.4 \mathrm{~nm}$ cutoff radii was applied. The nonbonded interactions in the range between these radii were updated every fifth time step. The time step was $2 \mathrm{fs}$, and the temperature was kept at $298.15 \mathrm{~K}$ using a Berendsen thermostat ${ }^{36}$ with a coupling time of 0.1 ps. All bonds were kept constant with the SHAKE algorithm ${ }^{37}$ using a relative geometrical tolerance of $10^{-4}$ in the condensed phase simulations and $10^{-8}$ in the in vacuo simulations. For calculating the heat of vaporization, two simulations had to be carried out, as described earlier. ${ }^{14,16}$ The constant volume simulations of the lipids at the experimental density were equilibrated for at least 500 ps, when necessary up to $2 \mathrm{~ns}$, until the average pressure converged. The atomic coordinates and velocities of the final system configuration were used to generate initial positions and velocities for the gas phase simulations. Each individual molecule was translated to a grid point $50 \mathrm{~nm}$ apart from the other mole- cules. This new starting configuration representing a sample in vacuo at room temperature with no intermolecular interactions (long-range cut-loff = $1.4 \mathrm{~nm}$ ) allows for faster averaging (100 ps) of the gas phase potential energy over the 512 molecules, instead of simulating one molecule over a 512 times longer time period. The difference between the potential energies in the gas phase and the liquid phase is assumed to be directly related to the heat of vaporization (apart from a term RT) because the in vacuo system is considered to be an ideal gas. A calculation of the virial of the equilibrated liquid provides the pressure of the system. With the large number of different molecules investigated an automated procedure ${ }^{38-40}$ for parametrization of the coefficients against the two key properties, heat of vaporization and pressure, was not feasible. Instead, every new parameter set was individually considered and modified, slowly progressing to a desirable degree of consistency. To test the parameters found with this procedure, simulations at 
constant pressure were additionally carried out to compare the average density against experimental values. The reference pressure was set to the experimental pressure, the pressure coupling time was set to $0.5 \mathrm{ps}$, and a value for the compressibility $\kappa_{T}$ was used as determined from separate constant volume simulations as follows. For each molecule, two constant volume simulations of the liquid were carried out at volumes smaller $\left(V_{1}\right)$ and larger $\left(V_{2}\right)$ than the experimental volume $V$. These provide the difference in pressures $\left(p_{1}\right.$ and $\left.p_{2}\right)$ observed upon the change of volume, which yields the isothermal compressibility $\kappa_{T}$ through eq. (1),

$$
\kappa_{T}=\frac{V_{1}-V_{2}}{V\left(p_{2}-p_{1}\right)}
$$

Correct reproduction of the free energies of solvation of molecules or their aggregates in water is crucial for any accurate model description of biomolecular systems. The calculation of the free energy of hydration of molecules by MD simulation is straightforward, for example, by using the thermodynamic integration method. ${ }^{41,42}$ A solute molecule that is interacting with the solvent molecules is in state A. When its nonbonded interactions with the solvent molecules are switched off, it is in state B. A thermodynamic coupling parameter $\lambda$ is used to smoothly change state $\mathrm{A}(\lambda=0)$ into state $\mathrm{B}(\lambda=1)$ and/or vice versa. Individual MD simulations are carried out at a number of $\lambda$ values in the range $[0,1]$ and the average of the derivative of the Hamiltonian with respect to $\lambda,\langle\partial H / \partial \lambda\rangle_{\lambda}$, is calculated for each $\lambda$ value. The free energy of hydration is then obtained by numerically integrating these averages from $\lambda=1$ to $\lambda=0 .{ }^{43}$

The solute-water systems for the thermodynamic integration calculations were set up as follows. A single aliphatic molecule was introduced into a truncated octahedron box with water (minimum solute to solvent distance of $0.23 \mathrm{~nm}$ ) such that the solute to wall distance was at least $1.6 \mathrm{~nm}$, or $1.75 \mathrm{~nm}$ if the solute molecule conformation had several kinks. This rather large solute to box-wall distance will allow the solute to expand without interacting with its own periodic image when periodic boundary conditions are applied. The number of water molecules in the box was always over a thousand. The simple point charge (SPC) water model was used. ${ }^{44}$ The solute-water configuration was energy minimized under periodic boundary conditions using the steepest descent method. Subsequently, the system was equilibrated for 200 ps of MD at room temperature. A reaction-field contribution originating from interactions beyond the long-range $(1.4 \mathrm{~nm})$ cutoff distance was added to the forces. ${ }^{45}$ A value of 66.6 was used for the dielectric permittivity $\varepsilon_{\mathrm{RF}}$ of the continuum outside the cutoff sphere, as recently estimated for the SPC model. ${ }^{46}$ After equilibration, a 1-ns slow growth simulation ${ }^{42}$ was performed as a means to obtain starting configurations and velocities for the simulations at fixed $\lambda$ values for thermodynamic integration. Initial thermodynamic integration free-energy values were obtained by numerical integration of $\langle\partial H / \partial \lambda\rangle_{\lambda}$ using 21 equally spaced $\lambda$ values between 0 and 1 (inclusive). Where the curvature of the integrand was large, additional $\lambda$ values were used. The simulation time was chosen in accordance to the rate of convergence of $\langle\partial H / \partial \lambda\rangle_{\lambda}$. Typically, $25 \lambda$ values were used with equilibration periods of $50 \mathrm{ps}$ and averaging periods of $150 \mathrm{ps}$, in some cases extended to 350 or even 950 ps. A soft-core $\lambda$-dependent interaction function was used to evaluate the solute-solvent van der Waals interactions in the slow growth and thermodynamic integration simulations, with a parameter $\alpha_{\mathrm{LJ}}$ of $0.5{ }^{14,35,47}$ Plots of the convergence of the free energy values as function of the number of $\lambda$ points and the extent of sampling (not shown) suggest that the uncertainty in the numbers shown in Table $\mathrm{V}$ (column 45A3) is in all cases below $0.5 \mathrm{~kJ} / \mathrm{mol}$.

\section{Results and Discussion}

The GROMOS force field makes use of the concept of charge groups. The atoms that belong to a charge group are chosen such that their partial atomic charges add up to zero (or to a multiple of \pm e). In the GROMOS nonbonded interaction subroutines the atom-atom nonbonded interactions are calculated for atom pairs within a charge group, and for atom pairs between charge groups. Considering two different charge groups, either all or none of the atom pairs for which one atom belongs to one charge group and the other atom belongs to the other charge group are contributing to the nonbonded force. This implies that the charge groups should be approximately spherical, and that if the largest charge groups in the system are of diameter $d$, the nonbonded cutoff radius $R_{c}$ must be chosen much larger than $d$ in order to avoid omission of atom pairs from neighboring charge groups in the calculation of the nonbonded forces. If charge groups in alkanes are too big, i.e., if the number of charge groups per alkane molecule is too small, the missing nonbonded interactions will be reflected in a too high pressure, because attractive van der 


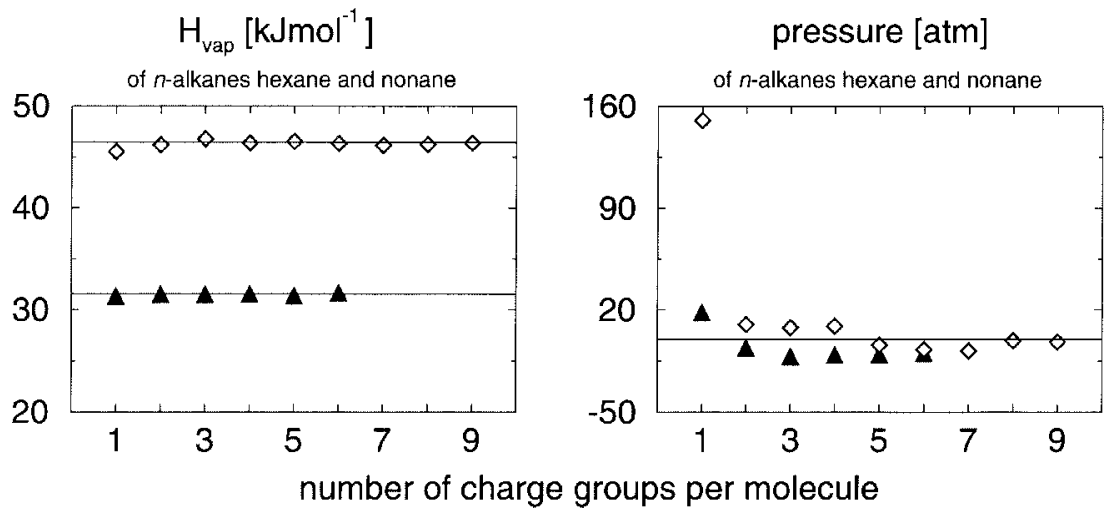

FIGURE 1. Heat of vaporization and pressure as function of the number of charge groups used. Simulated heat of vaporization and pressure in equilibrated liquid simulations (1 ns of 512 molecules) at constant volume and temperature for $n$-hexane $(\boldsymbol{\Delta})$ and $n$-nonane $(\diamond)$ as a function of the number of charge groups defined for each molecule. The solid horizontal lines represent the experimental values.

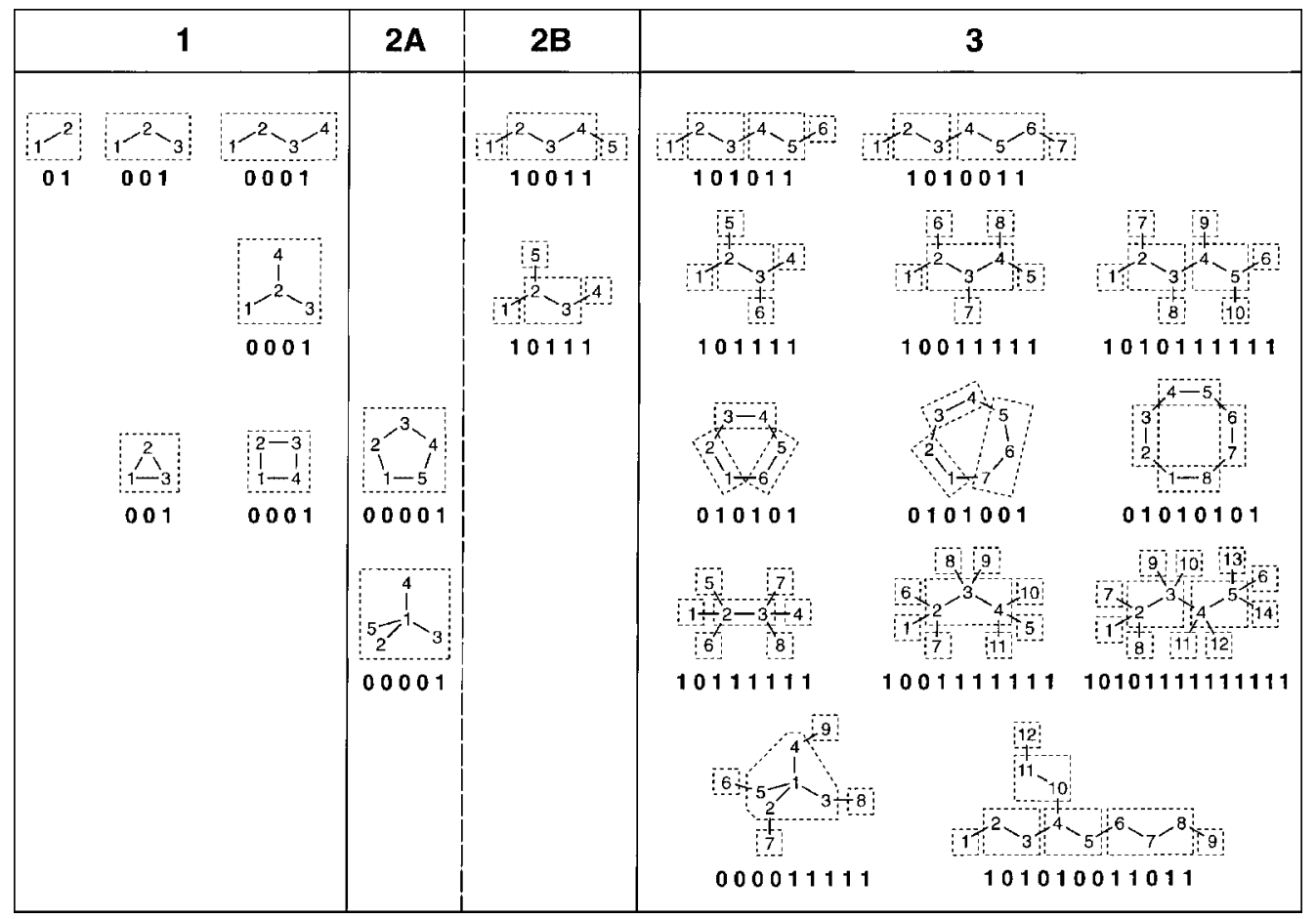

FIGURE 2. Examples of charge-group definition for a set of uncharged aliphatic molecules. Definition of charge group boundaries (dashed lines) as used in the simulations using the GROMOS force field. The examples of united-atom aliphatic hydrocarbon structures have been ordered according to the following set of rules: (1) if the total number of united atoms in a molecule is less or equal to 4 , use one charge group for the whole molecule; (2) if the total number of united atoms in a molecule or a molecule fragment is equal to 5, use: (A) if the maximal width of the molecule corresponds to the span of one bond angle, one charge group, (B) else follow rule (3) accordingly; (3) for molecules not subject to rules (1) and (2A), e.g., with more than five united atoms do take all $\mathrm{CH}_{3}$ united atoms into single charge groups. The remaining structure should be judged using rule (2), before the remaining fragments are divided into segments of two atoms per charge group and three atoms per charge group. The united atoms of each structure have been numbered and the charge-group codes $(0$ or 1$)$ of the corresponding GROMOS96-topology building blocks are displayed below each structure. 


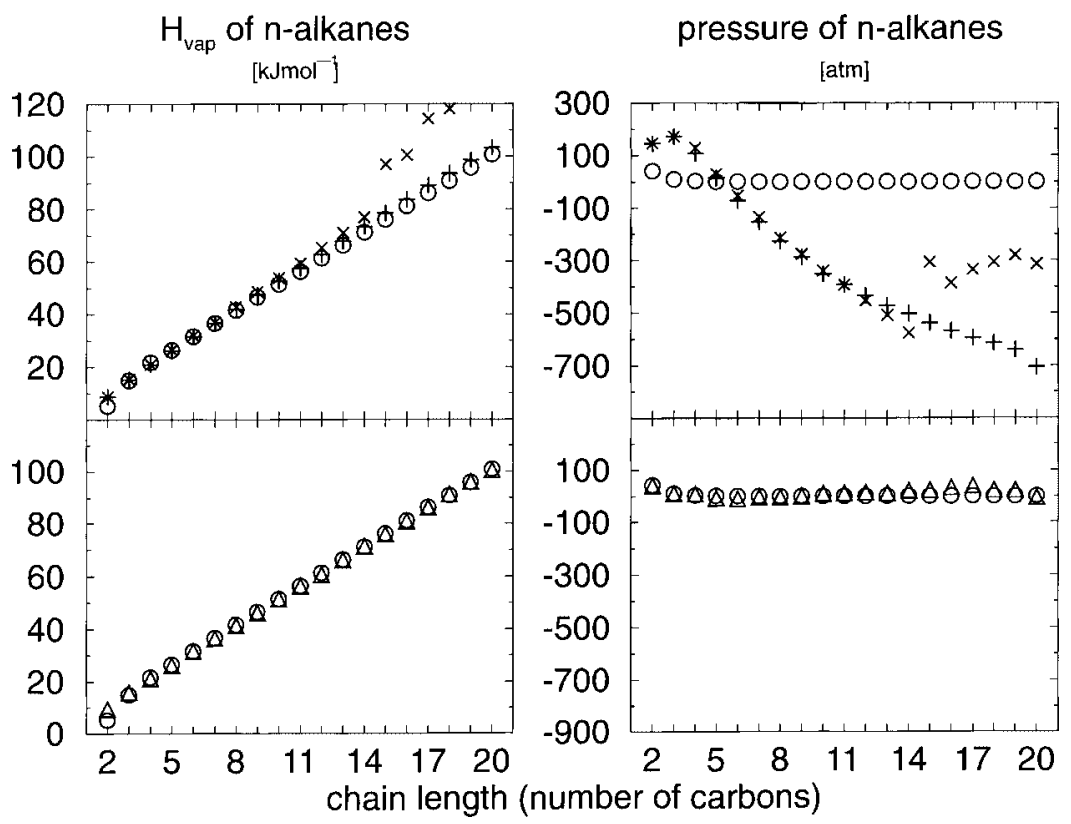

FIGURE 3. (top) Liquid key properties of $n$-alkanes using GROMOS96 liquid force fields $43 A 1^{13}$, 14 and $43 A 2 .{ }^{16}$ GROMOS96 force fields 43A1 $(\times)$ and 43A2 $(+)$ have been tested for a series of $n$-alkanes (ethane to eicosane). Whereas the heat of vaporization is close to experimental values $(O)$ for both force fields - with a slight improvement of 43A2 over 43A1 - the pressure in a constant volume simulation drastically drops below zero beyond a chain length of 6 ( $n$-hexane). The jump in simulated values obtained using force field set 43A1 occurs beyond chain length 14 , where these alkane structures crystallized after equilibration. (bottom) Results for the new set $45 \mathrm{~A} 3(\triangle)$. The van der Waals parameters of $\mathrm{CH}_{2}$ and $\mathrm{CH}_{3}$ united atoms have been successfully reparametrized to reproduce heat of vaporization and pressure at constant volume at room temperature.

Waals forces are then neglected. This is illustrated in Figure 1 for MD simulations of liquid $n$-hexane $(\boldsymbol{\Delta})$ and liquid $n$-nonane $(\diamond)$. In Figure 2 , the recommended charge group definitions for alkane molecules modeled using the GROMOS force field are displayed. A charge group contains at most five atoms, which are separated (except for $n$-butane) by at most two bonds. This charge group definition yields accurate results when used in conjunction with a $1.4 \mathrm{~nm}$ cutoff distance for nonbonded interactions. We note that the use of charge groups is computationally efficient even for a model in which atoms bear zero partial charge, because neighbor searching is faster for charge groups than for atoms, as long as there are charge groups containing more than one atom.

The performance of the GROMOS96 force-field parameter sets $43 \mathrm{~A} 1^{13,14}$ and $43 \mathrm{~A} 2^{16}$ with respect to MD simulation of single chain alkanes of length 2 to 20 in the condensed phase is shown in Figure 3 (top). The original GROMOS96 parameter set $43 \mathrm{~A} 1(\times)$ reproduces the experimental heat of vaporization of liquid $n$-alkanes $(\bigcirc)$ up to a chain length of about 14 and yields excellent agreement for $n$-alkanes of length 3 to 7 . The more recent pa- rameter set 43A2 $(+)$ yields good agreement up till a chain length of 20 . However, both parameter sets fail to reproduce the correct pressure in constant volume simulations at the experimental density of the liquids. This observation forced us to reconsider the van der Waals parameters for the $\mathrm{CH}_{2}$ and $\mathrm{CH}_{3}$ united atoms in the GROMOS force field. These atoms are abundant in systems composed of lipids and their parameters will affect the simulated density and energy of membranes. A reparametrization of the $\mathrm{CH}_{2}$ and $\mathrm{CH}_{3}$ van der Waals parameters for the whole set of $n$-alkanes led to a satisfactory result, as is shown in Figure 3 (bottom). The new set of parameters is called $45 \mathrm{~A} 3$. A detailed look at constant volume and constant pressure simulation results obtained with parameter set $45 \mathrm{~A} 3$ is offered in Table II. Three things are important to mention. First, for the smallest molecule, ethane, the united atom model used could not be parametrized to reproduce the density and heat of vaporization at the same time. Ethane always did evaporate after a finite simulation period at constant pressure conditions, and $\mathrm{CH}_{3}$ van der Waals parameters that reproduce both the experimental density and enthalpy could not be found. Second, in molecular dynamics simulation 
TABLE II.

n-Alkanes: A Comparison of Simulated and Experimental Data.

\begin{tabular}{|c|c|c|c|c|c|c|c|c|c|c|}
\hline \multirow{2}{*}{\multicolumn{2}{|c|}{ n-Alkane }} & \multirow{2}{*}{\multicolumn{2}{|c|}{$H_{\text {vap }}$}} & \multirow{2}{*}{\multicolumn{2}{|c|}{ Pressure }} & \multirow{3}{*}{$\frac{H_{\text {vap }}}{\text { NPT }}$} & \multirow{2}{*}{\multicolumn{2}{|c|}{ Volume }} & \multicolumn{2}{|c|}{ Compressibility } \\
\hline & & & & & & & & & \multirow{2}{*}{\multicolumn{2}{|c|}{$\begin{array}{l}\text { Exp } \\
10^{-2} \times \\
\left(\mathrm{kJmol}^{-1} \mathrm{~nm}^{-3}\right)^{-1}\end{array}$}} \\
\hline Name & $\begin{array}{l}\text { Number of } \\
\text { C-Atoms }\end{array}$ & $\begin{array}{c}\text { Exp } \\
\mathrm{kJ} \mathrm{mol}^{-1}\end{array}$ & $\begin{array}{c}\mathrm{NVT} \\
\mathrm{kJ} \mathrm{mol}^{-1}\end{array}$ & $\begin{array}{l}\text { Exp } \\
\text { atm }\end{array}$ & $\begin{array}{l}\text { NVT } \\
\text { atm }\end{array}$ & & $\begin{array}{l}\text { Exp } \\
n m^{3}\end{array}$ & $\begin{array}{l}\text { NPT } \\
\mathrm{nm}^{3}\end{array}$ & & \\
\hline Ethane & 2 & 5.16 & 9.50 & 41.08 & 41 & 14.88 & 81.16 & 82.25 & & 41.770 \\
\hline Propane & 3 & 14.79 & 15.79 & 9.48 & 9 & 15.89 & 76.05 & 75.99 & & 1.130 \\
\hline Butane & 4 & 21.62 & 21.19 & 2.43 & 8 & 21.16 & 86.24 & 86.38 & & 0.523 \\
\hline Pentane & 5 & 26.43 & 26.18 & 0.68 & -7 & 26.27 & 98.78 & 98.48 & 0.362 & 0.359 \\
\hline Hexane & 6 & 31.55 & 31.60 & 0.20 & -10 & 31.67 & 111.89 & 111.58 & 0.277 & 0.282 \\
\hline Heptane & 7 & 36.55 & 36.40 & 0.06 & -4 & 36.44 & 125.38 & 125.25 & & 0.241 \\
\hline Octane & 8 & 41.49 & 41.28 & 0.02 & -4 & 41.31 & 139.02 & 138.92 & 0.213 & 0.212 \\
\hline Nonane & 9 & 46.44 & 46.16 & 0.00 & 0 & 46.20 & 152.77 & 152.70 & 0.195 & 0.192 \\
\hline Decane & 10 & 51.37 & 51.52 & 0.00 & 10 & 51.48 & 166.53 & 166.68 & 0.182 & 0.182 \\
\hline Undecane & 11 & 56.33 & 56.31 & 0.00 & 11 & 56.30 & 180.44 & 180.51 & 0.171 & 0.169 \\
\hline Dodecane & 12 & 61.29 & 60.69 & 0.00 & 14 & 60.64 & 194.34 & 194.49 & 0.164 & 0.154 \\
\hline Tridecane & 13 & 66.23 & 66.18 & 0.00 & 10 & 66.14 & 208.25 & 208.47 & 0.157 & 0.142 \\
\hline Tetradecane & 14 & 71.17 & 71.43 & 0.00 & 19 & 71.33 & 222.17 & 222.58 & 0.151 & 0.148 \\
\hline Pentadecane & 15 & 76.15 & 75.97 & 0 & 22 & 75.70 & 236.11 & 236.75 & 0.146 & 0.144 \\
\hline Hexadecane & 16 & 81.09 & 80.84 & 0 & 32 & 80.72 & 250.06 & 250.61 & 0.142 & 0.142 \\
\hline Heptadecane & 17 & 86.19 & 86.03 & 0 & 38 & 85.75 & 263.94 & 264.66 & & 0.134 \\
\hline Octadecane & 18 & 90.79 & 91.26 & 0 & 22 & 90.52 & 277.73 & 278.81 & & 0.128 \\
\hline Nonadecane & 19 & 95.81 & 96.15 & 0 & 22 & 95.78 & 291.83 & 292.69 & & 0.132 \\
\hline Eicosane & 20 & 100.83 & 100.42 & 0 & -6 & 99.05 & 307.08 & 306.88 & & 0.142 \\
\hline
\end{tabular}

Columns four and six show the results from constant volume simulations with which the parametrization was undertaken. The third column shows experimental heat of vaporization to which simulated results in the fourth column can be compared. Corresponding experimental and simulated pressures are given in columns five and six. Columns seven through nine compare experiments with constant pressure simulations. Column seven contains the calculated head of vaporization at constant pressure to compare with columns three and four. The obtained density is reflected in columns eight and nine which contain the experimental and simulated volumes. All experimental data in columns three, five, and eigth was taken from ref. 54. For some molecules the experimental isothermal compressibility taken from CRC Handbook of Chemistry and Physics is given in column ten. Isothermal compressibility from simulations is shown in the last column. Except for the smaller $n$-alkanes indicated with two asterisks, these values have directly been used for the pressure coupling simulations. In the case of the smaller $n$-alkanes an increase of the pressure coupling time $\tau_{p}$ to $5 \mathrm{ps}$ instead of $0.5 \mathrm{ps}$ was necessary to keep the volume constant. However in the case of ethane (one asterisk), the liquid evaporated into the larger volume of $291.37 \mathrm{~nm}^{3}$ after about $100 \mathrm{ps}$.

the pressure is more sensitive than the density to changes in force-field parameters, i.e., parametrization based on fitting to the experimental pressure in constant volume simulations is more precise than one based on fitting to the experimental density in constant pressure simulations. In practice, if in a constant volume simulation the pressure deviates less than $100 \mathrm{~atm}$ from the experimental value (usually $1 \mathrm{~atm}$ ), the corresponding constant pressure simulation will closely reproduce the experimental density or volume. With this approach perfect agreement in volume could be achieved, with errors below $0.5 \%$ for most of the $n$-alkanes investigated. Third, at constant volume a slight increase of pressure with chain length is noticeable going from $n$-hexane to $n$-heptadecane, after which the pressure slightly decreases again. This may reflect the experimental observation that $n$-heptadecane should be liquid at room temperature (melting point $295 \mathrm{~K}$ ) whereas $n$-octadecane should be still solid (melting point $301 \mathrm{~K}$ ). In the literature another united atom model was found that was mainly used for C44 chains. ${ }^{48}$ The $\mathrm{CH}_{2}$ and $\mathrm{CH}_{3}$ parameters of our new GROMOS96 45A3 parameter set are closer to the values used in ref. 48 for polymer melts $(\sigma=$ $0.4 \mathrm{~nm}$ for both, $\varepsilon=0.389 \mathrm{~kJ} \mathrm{~mol}^{-1}$ for $\mathrm{CH}_{2}$ and $\varepsilon=0.947 \mathrm{~kJ} \mathrm{~mol}^{-1}$ for $\mathrm{CH}_{3}$ united atoms, compare to Table VI) than to the older $43 \mathrm{~A} 1$ or $43 \mathrm{~A} 2$ parameter values. The same is true when comparing the old and new GROMOS96 values to those $\left(\sigma=0.395 \mathrm{~nm}\right.$ and $\varepsilon=0.382 \mathrm{~kJ} \mathrm{~mol}^{-1}$ for $\mathrm{CH}_{2}$ and $\sigma=0.375 \mathrm{~nm}$ and $\varepsilon=0.815 \mathrm{~kJ} \mathrm{~mol}^{-1}$ for 
TABLE III.

Cyclic Alkanes: A Comparison between Simulated and Experimental Data.

\begin{tabular}{|c|c|c|c|c|c|c|c|c|c|c|}
\hline \multirow[b]{3}{*}{$\begin{array}{l}\text { Cycloalkane } \\
\text { Name }\end{array}$} & \multirow[b]{3}{*}{$\begin{array}{l}\text { Number of } \\
\text { C-Atoms }\end{array}$} & \multirow{2}{*}{\multicolumn{2}{|c|}{$H_{\text {vap }}$}} & \multirow{2}{*}{\multicolumn{2}{|c|}{ Pressure }} & \multirow{3}{*}{$\frac{H_{\text {vap }}}{\mathrm{NPT}}$} & \multirow{2}{*}{\multicolumn{2}{|c|}{ Volume }} & \multicolumn{2}{|c|}{ Compressibility } \\
\hline & & & & & & & & & \multirow{2}{*}{\multicolumn{2}{|c|}{$\begin{array}{c}\text { Exp NVT } \\
10^{-2} \times \\
\left(\mathrm{kJmol}^{-1} \mathrm{~nm}^{-3}\right)^{-1}\end{array}$}} \\
\hline & & $\begin{array}{c}\text { Exp } \\
\mathrm{kJ} \mathrm{mol}^{-1}\end{array}$ & $\begin{array}{c}\mathrm{NVT} \\
\mathrm{kJ} \mathrm{mol}^{-1}\end{array}$ & $\begin{array}{l}\text { Exp } \\
\text { atm }\end{array}$ & $\begin{array}{l}\text { NVT } \\
\text { atm }\end{array}$ & & $\begin{array}{l}\text { Exp } \\
n m^{3}\end{array}$ & $\begin{array}{l}\mathrm{NPT} \\
\mathrm{nm}^{3}\end{array}$ & & \\
\hline Cyclobutane & 4 & 23.64 & 21.71 & 1.57 & 816 & 19.38 & 69.24 & 78.33 & & 0.181 \\
\hline Cyclopentane & 5 & 28.53 & 27.72 & 0.42 & 36 & 26.56 & 80.47 & 84.24 & $0.221^{a}$ & 0.158 \\
\hline Cyclohexane & 6 & 33.05 & 33.25 & 0.13 & -0 & 33.42 & 92.45 & 91.74 & 0.196 & 0.182 \\
\hline Cycloheptane & 7 & 38.53 & 38.65 & 0.03 & +2 & 38.63 & 103.50 & 103.52 & $0.153^{a}$ & 0.150 \\
\hline Cyclooctane & 8 & 43.35 & 44.13 & 0.01 & -25 & 44.21 & 114.67 & 114.45 & $0.133^{a}$ & 0.134 \\
\hline
\end{tabular}

a Experimental values taken at $T=293 \mathrm{~K}$.

Cyclic alkanes: a comparison between simulated and experimental data. Analogously to Table II, experimental and simulated properties are given for a series of cycloalkanes in the liquid phase.

$\left.\mathrm{CH}_{3}\right)$ derived in ref. 49 for $n$-alkane phase equilibria.

The next step was to investigate cycloalkanes in a series from cyclobutane to cyclo-octane using the new $\mathrm{CH}_{2}$ parameters. The pressure at constant volume increased to about $1000 \mathrm{~atm}$ for all cycloalkanes. This meant that either $\mathrm{CH}_{2}$ parameters had to be compromised to fit experimental data for $n$-alkanes and cycloalkanes, thereby loosing the excellent agreement for the more relevant $n$-alkanes, or a new $\mathrm{CH}_{2}$ united atom type for cycloalkanes had to be introduced, as had already been done earlier for $\mathrm{CH}$ groups in aromatic rings. ${ }^{13}$ The latter option was chosen, and an atom type 44 named $\mathrm{CH} 2 \mathrm{r}$ ( $\mathrm{r}$ for ring) was introduced in the GROMOS96 force field. Table III shows some properties of cycloalkanes obtained with the optimized parameter set $45 \mathrm{~A} 3$. Our aim was to achieve good agreement with experiment primarily for cyclohexane, the most common species and widely used as organic solvent. ${ }^{50-52}$ It was possible to keep the pressure and volume close to the experimental values for cyclohexane and larger rings, but not for the smaller ones. The geometry of smaller rings is unfavorable for the dihedral angle potentials (type 17) and bond-angle bending potentials (type 14) used (Table I). Thus, relative to the experimental values, in the simulations at constant pressure the densities of cyclopentane and cyclobutane were off by 5 and $12 \%$, respectively, and the heats of vaporization were off by 3 and $8 \%$, respectively. The $45 \mathrm{~A} 3$ parameter set will be applicable to most mixed systems of linear and cyclic alkanes.

The next step was to consider branched alkanes, for example, isoalkanes and neoalkanes that contain two other (united) atom types, $\mathrm{CH}_{1}$, and
$\mathrm{CH}_{0}$. The latter has been introduced as a new atom type 45 , because the bare carbon with atom type 11 was initially designed for use in planar groups, for example, carbonyl and aromatic groups, and not for tetrahedral carbons. Table IV shows species by their name and the atom types involved, together with simulated and experimental properties. Some of the selected species are of more relevance regarding their occurrence as structural elements in biomolecular systems than others. In such systems methylated branches occur as in isoalkanes. 4-Propylnonane and 6-methylundecane have larger branches, and were chosen as test molecules because they have been used as actual lipid tails in experiments ${ }^{53}$ and because they combine the $\mathrm{CH}_{1}$ center with shorter and longer chains. The species isobutane, isopentane, 6-methylundecane, and 4-propylnonane, which show increasing interactions of the $\mathrm{CH}_{1}$ central atom with $\mathrm{CH}_{2}$ instead of $\mathrm{CH}_{3}$, show properties in close agreement to the experimental data. The series isobutane, 2,3-dimethylbutane, 2,3,4-trimethylpentane, 2,3(R),4(S),5-tetramethylhexane covers the interaction between $\mathrm{CH}_{1}$ and $\mathrm{CH}_{3}$ united atoms. It is noticeable how the pressure is reduced towards longer chains. It was impossible to find a parameter set that does not show this trend without having bad side effects on interactions of other atoms $\left(\mathrm{CH}_{2}\right.$, $\mathrm{CH}_{0}$ ) with $\mathrm{CH}_{1}$ atoms. It is known from experiment that tetramethylhexane should not solidify at room temperature. It does not in our simulations either, but the observed drop in pressure with increasing chain length indicates that this process might have started. However, experimental results given in ref. 54 for tetramethylhexane do not mention that it consists of a racemic mixture of two 


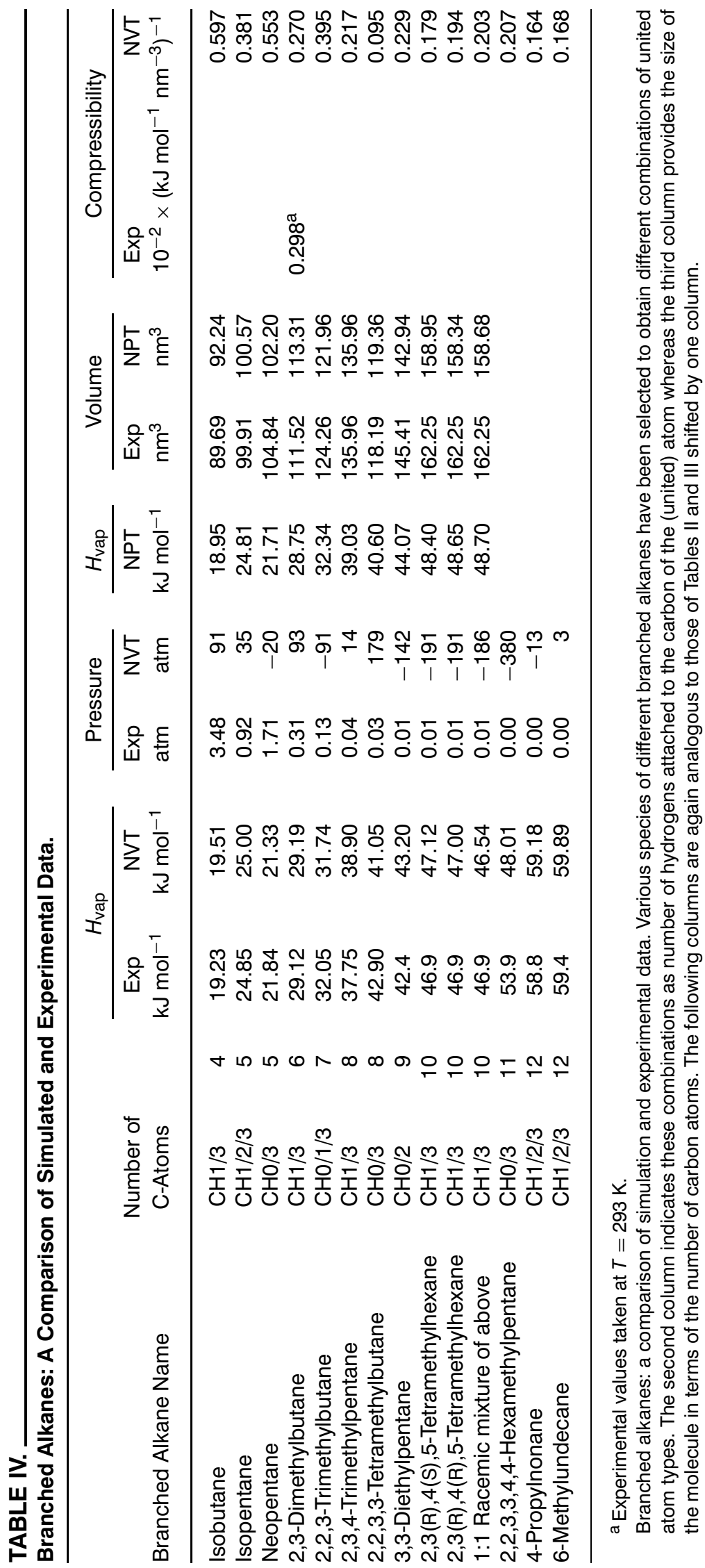


diastereomers. Three independent simulations were performed to check whether the RS and the RR compounds are different in their properties or if the racemic mixture $(1: 1$ in a first approximation because no synthesis description indicative of a particular distribution was found) gives results closer to the experimental properties with the chosen parameter set. After a long equilibration of $1.5 \mathrm{ns,}$ differences in the liquid properties are negligible, and this might explain why none of the experimental reports mentions stereospecificity. Combinations of a tetrahedral carbon and $\mathrm{CH}_{3}$ groups occur in the series neopentane, tetramethylbutane, and hexamethylpentane. Here, again, the smaller molecules fit the experimental properties much more easily than the larger one. Hexamethylpentane is a solid at room temperature, it melts at $64-66^{\circ} \mathrm{C}^{55}$ which is reflected in the large negative pressure in the constant volume simulation. A fully methylated chain is rare in nature, and therefore, of less relevance in biomolecular simulations. Further investigations might consider parameters of dihedral angle torsional potentials involving $\mathrm{CH}_{1}$ and $\mathrm{CH}_{0}$ tetrahedral carbon atoms to improve the properties of this series, in the same way as the change from the GROMOS96 43A1 parameter set to the $43 \mathrm{~A} 2$ parameter set involved a substantial improvement for dihedral potentials of $n$-alkane chains. Finally, a combination of the $\mathrm{CH}_{0}$ carbon with $\mathrm{CH}_{2}$ and $\mathrm{CH}_{1}$ united atoms was tested by considering 3,3-diethylpentane and 2,2,3trimethylbutane, respectively. Surprisingly, while tetramethylbutane is a solid at room temperature, the related 2,2,3-trimethylbutane is liquid. Indeed, we see this feature confirmed in our simulations with the new force-field parameters. The $\mathrm{CH}_{1}$ parameters of our new GROMOS96 45A3 parameter set are closer to the values derived in ref. 49 for isobutane phase equilibria $(\sigma=0.465 \mathrm{~nm}$ and $\varepsilon=$ $0.0831 \mathrm{~kJ} \mathrm{~mol}^{-1}$ ) than to the older $43 \mathrm{~A} 1$ or $43 \mathrm{~A} 2 \mathrm{pa}-$ rameter values.

After the 45A3 parameter set had been obtained it still had to prove its applicability to mixed water/solute systems. Therefore, free energies of hydration were calculated for a number of selected compounds, as described in the Methods section. GROMOS96 uses different van der Waals $C_{12}$ parameters for the oxygen atom types OW of SPC water, depending on whether it interacts with an apolar or a polar (hydrogen bond donor or acceptor) atom. Thus, in a mixed alkane-water system the $\mathrm{C}_{12}^{1 / 2}(1)$ of $\mathrm{OW}$ is used for interactions with $\mathrm{CH}_{n}$ atoms and the $C_{12}^{1 / 2}(2)$ is used for water-water interactions. Because of the changes made in the van der Waals parameters of the aliphatic groups, the
$C_{12}^{1 / 2}(1)$ of OW had to be reparametrized (Table VI) to reproduce the experimental free energies of hydration of the set of test alkanes. Interestingly, the new value of the $\mathrm{C}_{12}^{1 / 2}(1)$ parameter of $\mathrm{OW}$ is equal to its $\mathrm{C}_{12}^{1 / 2}(2)$ value (Table VI), which improves the transferability of the model. The new force field $45 \mathrm{~A} 3$ reproduces not only the free energies of hydration of $n$-alkanes excellently, but also that of other cyclic or branched species (Table V). Specifically, the somewhat more moderate performance for cycloalkanes may be explained with the many energetically different conformations adopted by these molecules in the experiment, which are not sampled sufficiently by one solute molecule in water within finite simulation time. It can be assumed that the general and good agreement with the experiment will provide manyfold advantages for studying mixed systems of apolar substances with water, micellar, or other lipid aggregates in water, or inverted micelles in apolar solvents.

\section{Conclusions}

Since GROMOS96 was developed, several suggestions for changes to the force field have been made. ${ }^{56-58}$ All of them affect only small molecules or have little impact on biomolecular simulations of peptides and proteins. The changes suggested here are more dramatic, because they consider all (hydro)carbon (united) atoms present in the force field of GROMOS96 except the aromatic $\mathrm{CH}$ united atom CR1 (atom type 16) that was used for benzene and other aromatic rings. The changes in the GROMOS96 force field introduced with the parameter set $45 \mathrm{~A} 3$ will affect every molecule containing aliphatic atoms (types 11 through 14). The differences between parameter sets $45 \mathrm{~A} 3,43 \mathrm{~A} 2{ }^{16}$ and $43 \mathrm{~A} 1^{14}$ are summarized in Table VI. The $\mathrm{CH}_{2}$ and $\mathrm{CH}_{3}$ united atom parameters were optimized for liquid $n$-alkanes and kept fixed throughout the rest of the parametrization procedure. A new $\mathrm{CH}_{2}$ atom type, $\mathrm{CH} 2 \mathrm{r}$, had to be introduced to reproduce the

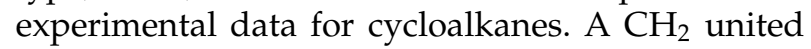
atom that would be appropriate for simulation of both $n$-alkanes and cycloalkanes could not be modeled. Finally, branched alkanes led to new $\mathrm{CH}_{1}$ parameters and to a new $\mathrm{CH}_{0}$ atom type (aliphatic bare carbon), which were used in combination with the previously derived $\mathrm{CH}_{2}$ and $\mathrm{CH}_{3}$ parameters.

Most force field development for classical molecular dynamics is nowadays either based on ab initio quantum-mechanical calculations or on experimental properties of the molecules investigated, or on a 
TABLE V.

Free Energies of Hydration for Varios Alkanes with Old and New Force-Field Parameters.

\begin{tabular}{|c|c|c|c|c|c|c|}
\hline \multirow{2}{*}{$\begin{array}{l}\text { Aliphatic } \\
\text { Compounds }\end{array}$} & \multirow{2}{*}{$\begin{array}{l}\text { Number of } \\
\text { C-Atoms }\end{array}$} & \multicolumn{5}{|c|}{ Free Energies of Hydration [kJ mol $\left.{ }^{-1}\right]$} \\
\hline & & Ref. 58 & Ref. 59 & Aver. & $43 \mathrm{~A} 1$ & $45 \mathrm{~A} 3$ \\
\hline Methane & 1 & 8.37 & 8.08 & 8.2 & 8.0 & 6.2 \\
\hline Ethane & 2 & 7.66 & 7.40 & 7.5 & 9.2 & 7.4 \\
\hline Propane & 3 & 8.18 & 8.26 & 8.2 & 9.0 & 8.6 \\
\hline Butane & 4 & 8.70 & 8.99 & 8.8 & 7.7 & 8.7 \\
\hline Pentane & 5 & 9.76 & 9.78 & 9.8 & - & 10.2 \\
\hline Hexane & 6 & 10.40 & 10.68 & 10.5 & - & 11.5 \\
\hline Heptane & 7 & 10.96 & 10.99 & 11.0 & - & 11.8 \\
\hline Octane & 8 & 12.10 & 12.08 & 12.1 & - & 13.0 \\
\hline Nonane & 9 & $12.58^{\mathrm{a}}$ & $12.76^{\mathrm{a}}$ & $12.7^{\mathrm{a}}$ & - & 12.6 \\
\hline Cyclobutane & 4 & $4.06^{\mathrm{a}}$ & - & $4.1^{\mathrm{a}}$ & - & 6.8 \\
\hline Cyclopentane & 5 & 5.02 & 5.06 & 5.0 & - & 5.1 \\
\hline Cyclohexane & 6 & 5.14 & 5.18 & 5.2 & - & 2.9 \\
\hline Cycloheptane & 7 & 3.33 & 3.36 & 3.3 & - & 4.4 \\
\hline Cyclooctane & 8 & 3.58 & 3.51 & 3.5 & - & 5.1 \\
\hline Isobutane & 4 & 9.70 & 9.55 & 9.6 & 10.4 & 10.3 \\
\hline Isopentane & 5 & 9.97 & 9.97 & 10.0 & - & 11.4 \\
\hline Neopentane & 5 & 10.46 & 11.22 & 10.8 & - & 9.9 \\
\hline
\end{tabular}

\footnotetext{
a Estimate from experimental fits of ref. 58, resp. ref. 59.

Free energies of hydration for various alkanes with old and new force field parameters. Three sections of aliphatic hydrocarbons contain a selection of the molecules presented in Tables II, III and IV, so $n$-alkanes, cycloalkanes and some branched alkanes. Their names are presented in the first and their number of carbons in the second column. Free energies of hydration have been measured (or calculated) by Cabani et al. ${ }^{59}$ (column 3) and Ben-Naim and Marcus ${ }^{60}$ (column 4) for which an average up to one decimal precision is given in column 5 . Free energies of hydration obtained using the original GROMOS96 force field $43 A 1$ published in ref. 14 are presented in column 6 and the values for the force field 45A3 are presented in column 7.
}

combination of both. The task of parametrization of a force field is very time consuming, not because the systems investigated are large but because the number of systems that have to be investigated is large. A larger calibration set of molecules (or pure systems) and their corresponding properties, to which the chosen parameters are fitted, will always lead to a more general force field than a smaller calibration set. The well-balanced GROMOS96 force-field parameter set 43A1, which produces fine results for a variety of biomolecules, did fail for long (more than 6 carbons) $n$-alkanes. It is, therefore, recommended to include at least one or a few long-chain species in the calibration set.

With this philosophy in mind, it was shown that the new $45 \mathrm{~A} 3$ parameter set perfectly fits $n$-alkane species longer than propane. A limitation of the force field lies in the very small $n$-alkanes and cycloalkanes. The essential features of these molecules are not captured in a united-atom model and harmonic bond-angle bending potentials may be insufficient to describe, for example, the ring puckering of cyclopentane. Properties of larger cycloalkanes are reproduced very well with the new GROMOS96 atom CH2r (type 44), especially those of cyclohexane. Properties of smaller branched aliphatics or larger aliphatics with few branches are also reproduced well. Only for highly methylated longer chains it seems difficult to come close to experimental densities and energies.

Finally, has been shown that after reparametrization of the van der Waals $C_{12}^{1 / 2}(1)$ oxygen (OW) parameter of the SPC water model for interactions with nonpolar atoms, the experimental free energies of hydration of alkanes are reproduced within the limits of accuracy of the thermodynamic integration method.

\section{Acknowledgment}

We would like to thank Peter Tieleman for testing lipid bilayer systems with the improved parameter set GROMOS96 43A2. It was his criticism that led us further investigate $n$-alkanes and other aliphatics. 
TABLE VI.

Changes in GROMOS96 Force-Field Parameters for Aliphatic Hydrocarbons.

\begin{tabular}{|c|c|c|c|c|c|c|c|}
\hline \multirow[b]{2}{*}{ TYPE } & \multirow{2}{*}{$\begin{array}{l}\text { Water } \\
\text { OW }\end{array}$} & \multicolumn{3}{|c|}{ Aliphatic Hydrocarbon Chains } & \multirow{2}{*}{$\begin{array}{l}\text { Methane } \\
\mathrm{CH} 4\end{array}$} & \multirow{2}{*}{$\begin{array}{l}\text { Aliphatic Rings } \\
\qquad \mathrm{CH} 2 \mathrm{r}\end{array}$} & \multirow{2}{*}{$\begin{array}{c}\text { Tetrahedral Aliphatic C } \\
\mathrm{CHO}\end{array}$} \\
\hline & & $\mathrm{CH} 1$ & $\mathrm{CH} 2$ & $\mathrm{CH} 3$ & & & \\
\hline IAC & 4 & 12 & 13 & 14 & 15 & 44 & 45 \\
\hline \multicolumn{8}{|l|}{$C_{6}^{1 / 2}$} \\
\hline $43 \mathrm{~A} 1$ & 0.05116 & 0.06148 & 0.08429 & 0.09958 & 0.1148 & - & - \\
\hline $43 \mathrm{~A} 2$ & 0.05116 & 0.06148 & 0.08429 & 0.09958 & 0.1148 & - & - \\
\hline $45 \mathrm{~A} 3$ & 0.05116 & 0.07790 & 0.08642 & 0.09805 & 0.1148 & 0.08564 & 0.04896 \\
\hline \multicolumn{8}{|l|}{$\mathrm{C}_{12}(1)^{1 / 2}$} \\
\hline $43 A 1$ & 1.544 & 3.373 & 5.077 & 5.794 & 5.862 & - & - \\
\hline $43 \mathrm{~A} 2$ & 1.544 & 3.373 & 5.077 & 5.794 & 5.862 & - & - \\
\hline $45 \mathrm{~A} 3$ & 1.623 & 9.850 & 5.828 & 5.162 & 5.862 & 5.297 & 14.330 \\
\hline \multicolumn{8}{|l|}{$\mathrm{C}_{12}(2)^{1 / 2}$} \\
\hline $43 \mathrm{~A} 1$ & 1.623 & 3.373 & 5.077 & 5.794 & 5.862 & - & - \\
\hline $43 \mathrm{~A} 2$ & 1.623 & 3.373 & 5.077 & 5.794 & 5.862 & - & - \\
\hline $45 \mathrm{~A} 3$ & 1.623 & 9.850 & 5.828 & 5.162 & 5.862 & 5.297 & 14.330 \\
\hline \multicolumn{8}{|l|}{$\mathrm{CS}_{6}^{1 / 2}$} \\
\hline $43 \mathrm{~A} 1$ & 0.05116 & 0.05396 & 0.06873 & 0.08278 & 0.1148 & - & - \\
\hline $43 \mathrm{~A} 2$ & 0.05116 & 0.05396 & 0.06873 & 0.08278 & 0.1148 & - & - \\
\hline $45 \mathrm{A3}$ & 0.05116 & 0.05396 & 0.06873 & 0.08278 & 0.1148 & 0.06873 & 0.04838 \\
\hline \multicolumn{8}{|l|}{$\mathrm{CS}_{12}^{1 / 2}$} \\
\hline $43 \mathrm{~A} 1$ & 1.544 & 1.933 & 2.667 & 3.473 & 5.862 & - & - \\
\hline $43 \mathrm{~A} 2$ & 1.544 & 1.933 & 2.178 & 2.456 & 5.862 & - & - \\
\hline $45 \mathrm{~A} 3$ & 1.623 & 1.933 & 2.178 & 2.456 & 5.862 & 2.178 & 1.837 \\
\hline \multicolumn{8}{|l|}{$\varepsilon$} \\
\hline $43 \mathrm{~A} 1$ & 0.7184 & 0.3139 & 0.4896 & 0.7323 & 1.2636 & - & - \\
\hline $43 \mathrm{~A} 2$ & 0.7184 & 0.3139 & 0.4896 & 0.7323 & 1.2636 & - & - \\
\hline $45 \mathrm{~A} 3$ & 0.6502 & 0.09489 & 0.4105 & 0.8672 & 1.2636 & 0.4793 & 0.006995 \\
\hline \multicolumn{8}{|l|}{$\sigma$} \\
\hline $43 \mathrm{~A} 1$ & 0.3113 & 0.3800 & 0.3920 & 0.3875 & 0.3710 & - & - \\
\hline 43A2 & 0.3113 & 0.3800 & 0.3920 & 0.3875 & 0.3710 & - & - \\
\hline $45 \mathrm{~A} 3$ & 0.3166 & 0.5019 & 0.4070 & 0.3748 & 0.3710 & 0.3955 & 0.6639 \\
\hline
\end{tabular}

GROMOS96 force field parameters are presented for water oxygen (OW) and aliphatic hydrocarbon united atoms $\left(\mathrm{CH}_{n}\right)$ from left to right. The standard $\mathrm{C}_{6}, \mathrm{C}_{12}$, and special third neighbour (1-4) $\mathrm{CS}_{6}, \mathrm{CS}_{12}$ parameters are used within GROMOS96 parameter files and presented in the first five sections. Parameters $\mathrm{C}_{6}{ }^{1 / 2}$ in $\left[\mathrm{kJ} \mathrm{mol}^{-1} \mathrm{~nm}^{6}\right]^{1 / 2}, \mathrm{C}_{12} 2^{1 / 2}$ in $10^{-3} \times\left[\mathrm{kJ} \mathrm{mol}^{-1} \mathrm{~nm}^{12}\right]^{1 / 2}$ for all sets $43 \mathrm{~A} 1$ to $45 \mathrm{~A} 3$. Additionaly the corresponding $\varepsilon$ and $\sigma$ parameters for the same Lennard-Jones potentials are given in the last two sections for the convenient comparison with other force fields. $\varepsilon$ and $\sigma$ values are converted from $\mathrm{C}_{12}(1)^{1 / 2}$ and $\mathrm{C}_{6}^{1 / 2}$ values and given in [ $\mathrm{kJ} \mathrm{mol}^{-1}$ ] and [nm], respectively. Every section consists of three rows providing values of the GORMOS96 force fields used. Set $A 1$ is the original set 43A1 provided since 1996. ${ }^{13,14}$ Set A2 (43A2) was published in ref. 16 and contained improved dihedral angle energy potentials and 1-4 van der Waals parameters for liquid $n$-alkanes, whereas set $A 3$ (45A3) is the one presented here considering a larger set of aliphatic hydrocarbons for parametrization against liquid properties.

\section{References}

1. Hünenberger, P. H.; van Gunsteren, W. F. Empirical Classical Interaction Functions for Molecular Simulation, in Computer Simulation of Biomolecular Systems, Theoretical and Experimental Applications; van Gunsteren, W. F.; Weiner,
P. K.; Wilkinson, A. J., Eds.; Kluwer Academic Publishers: Dordrecht, 1997; p. 3, vol 3.

2. Levitt, M. J Mol Biol 1974, 82, 393.

3. Levitt, M.; Hirshberg, M.; Sharon, R.; Daggett, V. Comput Phys Com 1995, 91, 215.

4. Hagler, A. T.; Huler, E.; Lifson, S. J Am Chem Soc 1974, 96, 5319. 
5. Dauber-Osguthorpe, P.; Roberts, V. A.; Osguthorpe, D. J.; Wolff, J.; Genest, M.; Hagler, A. T. Proteins Struct Funct Genet 1988, 4, 31.

6. Brooks, B. R.; Bruccoleri, R. E.; Olafson, B. D.; States, D. J.; Swaminathan, S.; Karplus, M. J Comput Chem 1983, 4, 187.

7. MacKerell, Jr.; Bashford, A. D.; Bellott, M.; Dunbrack, Jr.; Evanseck, J. D.; Field, M. J.; Fischer, S.; Gao, J.; Guo, H.; Ha, S.; Joseph-McCarthy, D.; Kuchnir, L.; Kuczera, K.; Lau, F. T. K.; Mattos, C.; Micknick, S.; Ngo, T.; Nguyen, D. T.; Prodhom, B.; Reiher, W. E., III; Roux, B.; Schlenkrich, M.; Smith, J. C.; Stote, R.; Straub, J.; Watanabe, M.; WiorkiewiczKuczera, J.; Yin, D.; Karplus, M. J Phys Chem B 1998, 102, 3586.

8. Weiner, S. J.; Kollman, P. A.; Case, D. A.; Singh, U. C.; Ghio, C.; Alagona, G.; Profeta, S., Jr.; Weiner, P.; J Am Chem Soc 1984, 106, 765.

9. Cornell, W. D.; Cieplak, P.; Bayly, C. I.; Gould, I. R.; Merz, K. M., Jr.; Ferguson, D. M.; Spellmeyer, D. C.; Fox, T.; Caldwell, J. W.; Kollman, P. A. J Am Chem Soc 1995, 117, 5179.

10. Hermans, J.; Berendsen, H. J. C.; van Gunsteren, W. F.; Postma, J. P. M. Biopolymers 1984, 23, 1513.

11. van Gunsteren, W. F.; Berendsen, H. J. C. Groningen Molecular Simulation (GROMOS) Library Manual; BIOMOS b.v., Groningen, 1987.

12. Egberts, E.; Marrink, S. J.; Berendsen, H. J. C. Eur Biophys J $1994,22,423$.

13. van Gunsteren, W. F.; Billeter, S. R.; Eising, A. A.; Hünenberger, P. H.; Krüger, P.; Mark, A. E.; Scott, W. R. P.; Tironi, I. G. Biomolecular Simulation: The GROMOS96 Manual and User Guide; vdf Hochschulverlag AG an der ETH Zürich and BIOMOS b.v.: Zürich, Groningen, 1996.

14. Daura, X.; Mark, A. E.; van Gunsteren, W. F. J Comput Chem 1998, 19, 535.

15. van Gunsteren, W. F.; Daura, X.; Mark, A. E. Encyclopedia Comput Chem 1998, 2, 1211.

16. Schuler, L. D.; van Gunsteren, W. F. Mol Sim 2000, 25, 301.

17. Jorgensen, W. L.; Tirado-Rives, J. J Am Chem Soc 1988, 110, 1657.

18. Jorgensen, W. L.; Maxwell, D. S.; Tirado-Rives, J. J Am Chem Soc 1996, 118, 11225.

19. van Gunsteren, W. F.; Mark, A. E. J Chem Phys 1998, 108, 6109.

20. Daura, X.; Gademann, K.; Jaun, B.; Seebach, D.; van Gunsteren, W. F.; Mark, A. E. Angew Chem Int Ed 1999, 38, 236.

21. Peter, C.; Daura, X.; van Gunsteren, W. F. J Am Chem Soc $2000,122,7461$

22. van Gunsteren, W. F.; Bürgi, R.; Peter, C.; Daura, X. Angew Chem Int Ed 2001, 40, 351.

23. Bonvin, A. M. J. J.; van Gunsteren, W. F. J Mol Biol 2000, 296, 255.

24. van Gunsteren, W. F.; Berendsen, H. J. C.; Hermans, J.; Ho, W. G. J.; Postma, J. P. M. Proc Natl Acad Sci USA 1983, 80, 4315.

25. Berendsen, H. J. C.; van Gunsteren, W. F.; Zwinderman, H. R. J.; Geurtsen, R. G. Ann NY Acad Sci 1986, 482, 269.

26. Smith, P. E.; van Schaik, R. C.; Szyperski, T.; Wüthrich, K.; van Gunsteren, W. F. J Mol Biol 1995, 246, 356.

27. Smith, L. J.; Mark, A. E.; Dobson, C. M.; van Gunsteren, W. F. Biochem 1995, 34, 10918.
28. Brunne, R. M.; Berndt, K. D.; Güntert, P.; Wüthrich, K.; van Gunsteren, W. F. Proteins 1995, 23, 49.

29. Stocker, U.; van Gunsteren, W. F. Proteins 2000, 40, 145.

30. Koehler, J. E. H.; Saenger, W.; van Gunsteren, W. F. Eur Biophys J 1987, 15, 211.

31. Koehler, J. E. H.; Saenger, W.; van Gunsteren, W. F. Eur Biophys J 1988, 16, 153.

32. Mark, A. E.; van Helden, S. P.; Smith, P. E.; Janssen, L. H. M.; van Gunsteren, W. F. J Am Chem Soc 1994, 116, 6293.

33. van Gunsteren, W. F.; Berendsen, H. J. C.; Geurtsen, R. G.; Zwinderman, H. R. J. Ann NY Acad Sci 1986, 482, 287.

34. Bonvin, A. M. J. J.; Sunnerhagen, M.; Otting, G.; van Gunsteren, W. F. J Mol Biol 1998, 282, 859.

35. Scott, W. R. P.; Hünenberger, P. H.; Tironi, I. G.; Mark, A. E.; Billeter, S. R.; Fennen, J.; Torda, A. E.; Huber, T.; Krüger, P.; van Gunsteren, W. F. J Phys Chem A 1999, 103, 3596.

36. Berendsen, H. J. C.; Postma, J. P. M.; van Gunsteren, W. F.; DiNola, A.; Haak, J. R. J Chem Phys 1984, 81, 3684.

37. Ryckaert, J. P.; Ciccotti, G.; Berendsen, H. J. C. J Comput Phys 1977, 23, 327.

38. Njo, S. L.; van Gunsteren, W. F.; Müller-Plathe, F. J Chem Phys 1995, 102, 6199.

39. Müller-Plathe, F.; Liu, H.; van Gunsteren, W. F. Comput Polymer Sci 1995, 5, 89.

40. Faller, R.; Schmitz, H.; Biermann, O.; Müller-Plathe, F. J Comput Chem 1999, 20, 1009.

41. Straatsma, T. P.; Berendsen, H. J. C. J Chem Phys 1988, 89, 5876.

42. van Gunsteren, W. F.; Beutler, T. C.; Fraternali, F.; Küng, P. M.; Mark, A. E.; Smith, P. E. In Computer Simulation of Biomolecular Systems, Theoretical and Experimental Applications; van Gunsteren, W. F.; Weiner, P. K.; Wilkinson, A. J., Eds.; Escom Science Publishers: Leiden, 1993; p. 315, vol. 2.

43. Daura, X.; Hünenberger, P. H.; Mark, A. E.; Querol, E.; Avilés, F. X.; van Gunsteren, W. F. J Am Chem Soc 1996, 118, 6285.

44. Berendsen, H. J. C.; Postma, J. P. M.; van Gunsteren, W. F.; Hermans, J. In Intermolecular Forces; Pullman, B., Ed.; Reidel: Dordrecht, 1981; p. 331

45. Tironi, I. G.; Sperb, R.; Smith, P. E.; van Gunsteren, W. F. J Chem Phys 1995, 102, 5451.

46. Glättli, A. Diploma thesis at the Laboratory of Physical Chemistry, ETH, Zürich, 2000.

47. Beutler, T. C.; Mark, A. E.; van Schaik, R. C.; Gerber, P. R.; van Gunsteren, W. F. Chem Phys Lett 1994, 222, 529.

48. Paul, W.; Yoon, D. Y.; Smith, G. D. J Chem Phys 1995, 103, 1702.

49. Wick, C. D.; Martin, M. G.; Siepmann, J. I. J Phys Chem B 2000, 104, 8008.

50. Tamura, H.; Yoshida, M.; Kusakabe, K.; Young-Mo, C.; Miura, R.; Kubo, M.; Teraishi, K.; Chatterjee, A.; Miyamoto, A. Langmuir 1999, 15, 7816.

51. Schmitz, H.; Faller, R.; Müller-Plathe, F. J Phys Chem B 1999, 103, 9731.

52. Price, W. S.; Soderman, O. J Phys Chem A 2000, 104, 5892

53. Ravoo, B. J.; Engberts, J. F. B. N. Langmuir 1994, 10, 1735.

54. TRC Thermodynamic Tables-Hydrocarbons; Texas A \& M University System: College Station, TX. 


\section{SCHULER, DAURA, AND VAN GUNSTEREN}

55. Fitjer, L.; Scheuermann, H. J.; Klages, U.; Wehle, D.; Stephenson, D. S.; Binsch, G. Chem Ber 1986, 119, 1144.

56. Müller-Plathe, F. Mol Sim 1996, 18, 133.

57. Walser, R.; Mark, A. E.; van Gunsteren, W. F.; Lauterbach, M.; Wipff, G. J Chem Phys 2000, 112, 10450.
58. Juchli, D. Diploma thesis at the Laboratory of Physical Chemistry, ETH, Zürich, 2000.

59. Cabani, S.; Gianni, P.; Mollica, V.; Lepori, L. J Solut Chem 1981, 10, 563.

60. Ben-Naim, A.; Marcus, Y. J Chem Phys 1984, 81, 2016. 